\title{
FLAME PROPAGATION ALONG THE INTERFACE BETWEEN A GAS AND A REACTING MEDIUM
}

\author{
C. SANCHEZ TARIFA AND A. MUÑOZ TORRALBO
}

Instituto Nacional de Técnica Aeroespacial, "Esteban Terradas," Madrid, Spain

\begin{abstract}
Flame propagation process over the surface of a solid or liquid fuel (or oxidizer) in contact with a gaseous oxidizer (or fuel) is studied.

Flame propagation velocity is calculated by assuming that flame reaches a certain location when temperature at the fuel surface at that location reaches an ignition temperature. This assumption is correct when the time consumed for the heating process is large as compared with the time required for vaporization of the fuel, mixing, and combustion of the oxidizerfuel vapors mixture.

Heating of the fuel ahead of the flame is studied by considering radiation and convection heat-transfer mechanisms flame to fuel, and bidimensional heat conduction within the fuel.

The problem lies in solving a heat-balanee partial-differential equation in the solid or liquid fuel for which heat fluxes from the flame are the fundamental boundary conditions. An analytical solution is obtained by means of a boundary-layer approximation, and general results are given.
\end{abstract}

An experimental and theoretical application of the general study is performed for the case of liquid hydrocarbon fuels burning in air.

\section{Introduction}

There are many important problems related to the general process of flame propagation along the interface between a gaseous oxidizer (or gaseous fuel) and a liquid or solid fuel (or oxidizer).

The problem covers a very large field, including processes such as flame spreading in fires, flame propagation over the surface of liquid fuels burning in air, or flame spreading over the surface of thermoplastic fuels during the ignition process in hybrid rocket motors.

The problem of flame spreading in fres has been studied mostly experimentally, although some theoretical studies have also been performed. ${ }^{1}$

Flame spreading over the surface of liquid fuels burning in air have been studied by us, ${ }^{2}$ and some results will be given in the present work.

The problem of flame propagation over the surface of solid propellants during the ignition process in rocket motors is receiving considerably attention. However, practically all these studies refer to the case of composite or double-base monopropellants. ${ }^{3-5}$ This is a problem of a different nature, because the monopropellant does not need a gaseous oxidizer to support a flame. However, some conclusions of the present work might be applied to the study of the aforementioned process.

There is very little information on the problem of flame spreading over the surface of solid propellants in a gaseous oxidizer environment in connection with the ignition process in hybrid rockets. Horowith, ${ }^{5}$ performed several measurements on flame-spreading velocities over the surface of solid composite-propellant constituents (thermoplastic fuels in an oxidizing atmosphere and a solid oxidizer in a gaseous fuel environment), but these studies were performed as part of a research program on flame spreading over the surface of composite propellants.

The process of flame propagation is essentially the same in all the aforementioned processes. If it is assumed that no reactant atmosphere exists far ahead of the flame, the propagation process takes place through the following sequence of events; heating of the fuel ahead of the flame, vaporization of this fuel and mixing with the oxidizer, and finally, ignition and combustion of this gaseous mixture.

This over-all process is very complicated. The flame propagation speed will be controlled by the over-all time needed for all these processes to take place. However, if the time required for any one of the partial processes is large as compared with the time needed for the other processes, this partial process would control the propagation speed and then, the study of the over-all process might be essentially simplified.

Heating of the fuel ahead of the flame as it progresses is the first and most essential process of the flame propagation mechanism. 
Therefore, it is very important to know the flame propagation mechanism from flame to fuel and to study the time consumed for the heating process since it may control propagation speed in many cases. Nevertheless, there is little information on these problems.

It has been recognized, for example, that for large flames, as those existing in forest or urban fires, heat transfer through radiation is predominant. On the other hand, since flame emissivity decreases exponentially with its size, it is believed that convection must be the predominant heat-transfer mechanisms in most flame-spreading processes over the surface of igniting solid propellants. However, a general analytical study of this heat-transfer problem in connection with flame spreading has not yet been performed.

In the present work, we study the heating process of the fuel ahead of the flame until a certain ignition temperature is reached. This study considers both radiation and convection heat-transfer mechanisms, and the relative importance of these two heat-transfer models are analytically studied and discussed. Flamepropagation speeds are calculated by assuming that the flame reaches a certain location when the temperature at the surface of the liquid or solid fucl reaches a given ignition temperature.

Propagation speeds calculated in this way would be correct if the time required for vaporization, mixing, and combustion is small as compared with the time needed to heat the fuel up to the ignition temperature. It is also required that the heat consumed by the evaporation of the fuel and by the ignition of the gaseous mixture be small as compared to the heat consumed to raise the temperature of the fuel up to ignition conditions.

Hicks, ${ }^{6}$ used a similar assumption to study ignition in solid monopropellants. On the other hand, other investigators ${ }^{7}$ have assumed that ignition of some types of solid monopropellants occurs through a gas-phase reaction which controls the process.

For our case of a bipropellant combination, we have verified the assumption for the case of flame spreading over the surface of liquid fuels burning in air. It is also expected that heating of the fuel will control the propagation process over the surface of some thermoplastic fuels burning in oxygen, when they have high ignition temperatures.

In all cases, the present work is intended to be a contribution to the study of the heating process of the fuel ahead of the flame, establishing a method to study the relative influence of radiation and convection heat-transfer mechanisms.

\section{Fundamental Assumptions}

Theoretical model of the process will be based upon the following assumptions (Fig. 1):

1. Stationary conditions with respect to a coordinate system moving at the same speed as the flamefront.

2. The gaseous oxidizer will be supposed to be at rest ahead of the flame, and this flame will be assimilated to a surface of discontinuity for the velocity. (The present study has been restricted to the case of flame propagation in a quiescent atmosphere. However, the study can be extended, as it is actually being done, to the case of a gas in motion.)

3. Heat transfer from flame to fuel ahead of the flame will be supposed to take place through radiation exclusively. Heat transfer from flame to fuel behind the flamefront will be assumed to take place through both convection and radiation.

Therefore, heat conduction from flame to fuel ahead of the flame is disregarded as compared to the heat transmitted through the solid or liquid fuel, since thermal conductivity of the fuel is much higher than thermal conductivity of the gas.

4. Flame velocity is given by condition that the flame reaches a certain location when the temperature of the fuel surface at that location
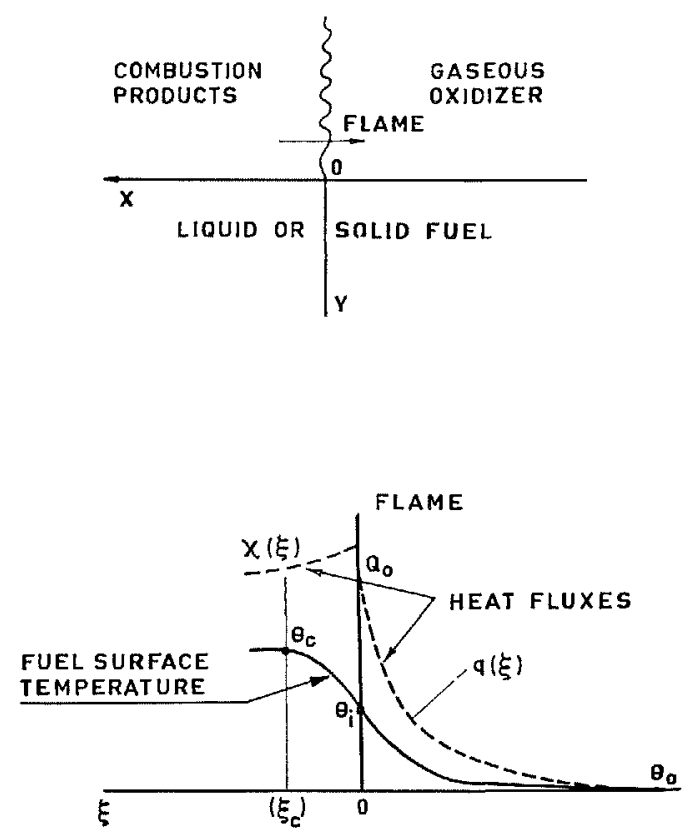

Frg. 1. Flame-propagation model. 
reaches a given ignition temperature. It will also be assumed that, after ignition, fuel-surface temperature keeps increasing until a constant combustion temperature is reached.

5. The problem will be initially studied assuming an infinite thickness of the fuel, although this assumption will be removed afterwards.

Under these assumptions the problem consists of solving a heat-transfer problem within the liquid or solid fuel, in which the heat fluxes reaching the fuel surface are the fundamental boundary conditions of the process."

\section{Fundamental Equations and Boundary Conditions}

Considering bidimensional conditions* within the fuel, the equation of energy with respect to a coordinate system of reference moving with the flame is

$\lambda\left(\partial^{2} T / \partial x^{2}\right)+\lambda\left(\partial^{2} T / \partial y^{2}\right)-\rho c u(\partial T / \partial x)=0$,

in which $u$ is the flame-propagation velocity.

A boundary-layer solution of this equation will be sought. Therefore, it will be expressed in integral form

$$
\begin{aligned}
\lambda(\partial / \partial x) \int_{0}^{\infty}(\partial T / \partial x) d y-\rho c u(\partial / \partial x) & \\
& \times \int_{0}^{\infty} T d y+Q_{0} \phi(x)=0
\end{aligned}
$$

in which $Q_{0} \phi(x)$ is the heat flux received by the fuel surface and transmitted through conduction within the fuel.

Introducing the dimensionless variables

$$
\begin{aligned}
\nu & =Q_{0} / \rho c T_{0} \\
\delta & =\lambda / \rho c \nu \\
\theta & =\left(T-T_{0}\right) / T_{0} \\
\xi & =x / \delta \\
\eta & =y / \delta,
\end{aligned}
$$

* The problem is presently being studied with axial symmetry.
Eq. (2) is written in the form

$$
\begin{aligned}
\left(\partial^{2} / \partial \xi^{2}\right) \int_{0}^{\infty} \theta d \eta- & (u / \nu)(\partial / \partial \xi) \\
& \times \int_{0}^{\infty} \theta d \eta+\phi(\xi)=0 .
\end{aligned}
$$

\section{Boundary Conditions}

a) Region $0 \geq \xi \geq-\infty$

Let $\phi(\xi)=q(\xi)$ be the dimensionless heat flux received by the fuel surface ahead of the flame, in which the influence of fuel reflectivity may be included. Assuming that this heat flux is absorbed at the fuel surface, and disregarding the radiant heat flux emitted by the fuel (the fuel surface temperature is low ahead of flame), the following boundary conditions result:

$\eta=0$

$$
\begin{aligned}
\frac{\partial \theta}{\partial \eta} & =-q(\xi) \\
\xi & =-\infty, \quad \theta=\theta_{0}=0
\end{aligned}
$$

$\eta \rightarrow \infty$

$$
\theta=\theta_{0}=0 \text {. }
$$

If the flame is assumed to have a rectangular shape, function $q(\xi)$ can be very well approximated by means of an exponential expression of $\xi$, as shown in Ref. 2.

\section{Region $0 \gtrless \xi ₹ \infty$}

Let $\phi(\xi)=\chi(\xi)$ be the net heat flux reaching the fuel surface and transmitted through conduction within the fuel. This heat flux results from the following balance:

$$
\chi(\xi)=\psi_{C}(\xi)+\psi_{R}(\xi)-\psi_{R S}\left(\xi, \theta_{s}\right)-\dot{m} q_{1},
$$

in which $\psi_{C}(\xi)$ and $\psi_{R}(\xi)$ are the heat transferred from the gas through convection and radiation, respectively: $\psi_{R S}\left(\xi, \theta_{s}\right)$ is the radiant heat emitted by the fuel surface; the term $\dot{m} q_{1}$ represents the heat consumed by the vaporization of the fuel ( $\dot{m}=$ evaporation rate, $q_{1}=$ latent heat of vaporization).

Convective heat transfer $\psi_{C}(\xi)$ may be approximated by assuming that behind the flame 
there is an uniform How of gas at flame temperature moving at a speed $u_{g}=\rho_{a} / \rho_{0} u$. Assuming laminar flow conditions, it may be taken approximately:

$$
Q_{0} \psi_{c}(\xi)=0.33 \bar{\lambda}_{g} P_{r g}^{1 / 2}\left(\rho_{\theta} u / \rho_{0_{\theta}} \nu_{0} \delta \xi\right)^{1 / 2} .
$$

Radiant heat transfer $\psi_{R}(\xi)$ can be taken as constant and equal to $q(0)$ in a region close to the flamefront. $\psi_{R S}(\xi)$ is easily calculated once the fuel surface temperature and fuel emissivity are known.

The term $\dot{m} q_{1}$ is very difficult to calculate because it corresponds to transient combustion conditions following ignition. Its value has been approximately calculated for liquids burning in air, ${ }^{8}$ but no information exists for the case of thermoplastic fuels burning in air or oxygen.

According to this and according to the fundamental assumptions, behind the flamefront the following boundary conditions exist

$\eta=0$

$$
\begin{aligned}
\partial \theta / \partial \eta & =-\chi(\xi) \\
\xi & =\xi_{c}, \quad \theta=\theta_{c} \\
(\partial \theta / \partial \xi)_{\xi=\xi_{c}} & =0
\end{aligned}
$$

$\eta \rightarrow \infty:$

$$
\xi \leq \xi_{c}, \quad \theta=\theta_{0}=0
$$

Finally, condition $\theta=\theta_{i}$ for $\xi=0$ gives the propagation velocity $u$, which is an eigenvalue of the differential equation.

\section{Solution of the Equation}

The present flame propagation process is especially suitable to be studied by means of a boundary-layer approximation, since only in a thin layer of the fuel will temperatures be modified by the propagation process, then a thermal boundary layer exists as will be verified.

This boundary-layer solution is obtained by approximating temperature profiles within the fuel by means of the exponential expression:

$$
\theta=\theta_{s} \exp \left\{-\left[\phi(\xi) / \phi_{s}\right] \eta\right\}
$$

Taking this expression into (8), the following ordinary differential equation results:

$$
\frac{d^{2} \Phi}{d \xi^{2}}-\frac{u}{\nu} \frac{d \Phi}{d \xi}+\phi(\xi)=0
$$

in which

$$
\Phi=\theta_{s}^{2} / \phi(\xi)
$$

Equation (16) is readily integrated once the expression of $\phi(\xi)$ is known, giving directly surface temperature $\theta_{s}(\xi)$. Introducing condition $\theta_{s}(0)=\theta_{i}$, an expression giving the dimensionless propagation speed $u / \nu$ is obtained.

This method of solution is valid for any expression of $\phi(\xi)$. In particular, the problem will be solved by taking

$\phi(\xi)=q(\xi)=\exp (\beta \xi)$

$$
(0 \geq \xi \geq-\infty)
$$

$\chi(\xi)=\chi=c t e$

$$
\left(0 \leq \xi \leq \xi_{0}\right)
$$

These values correspond, respectively, to an approximated expression for the radiant heat flux ahead of the flame, and to an average convective heat transfer behind the flame added to a constant radiant-heat flux.

Validity of the boundary-layer approximation is doubtful in a region far ahead of the flame in which the heat flux $\phi(\xi)$ is small, since in Eqs. (8) or (16) the first two terms will be predominant and, therefore, solution of the equation will tend to that of the one-dimensional case. This problem is solved by applying the boundarylayer solution only till a coordinate $\xi=\xi_{b}$ ahead of the flame at which $\phi\left(\xi_{b}\right)$ is small and solving Eq. (16) for $\phi(\xi)=0$ from $\xi=\xi_{b}$ to $\xi=-\infty$. At point $\xi=\xi_{b}$, both solutions are matched by equating temperatures and derivatives.

On the other hand, Eq. (16) is solved separately for regions $0 \geq \xi \geq \xi_{b}$, in which $\phi(\xi)=$ $\exp (\beta \xi)$, and region $0 \leq \xi \leq \xi_{c}$, in which $\phi(\xi)=\chi=$ cte. At $\xi=0$, both solutions are also matched.

Final result of all these calculations is given by the expressions

$$
\Psi \chi+\chi^{2}\left(\nu^{2} / u^{2}\right)\left[\log \frac{\chi^{2}\left(\nu^{2} / u^{2}\right)}{\chi^{\Psi}}-1\right]-\left(\theta_{c}{ }^{2}-\theta_{i}{ }^{2}\right)=0
$$


in which

$$
\begin{aligned}
\Psi & =\chi^{-1}(\nu / u)\left\{\frac{\theta_{i}{ }^{2}+\{\beta[\beta-(u / \nu)]\}^{-1}-2\{\beta[\beta-(u / \nu)]\}^{-1} \exp \left(\beta \xi_{b}\right)}{1-[\beta-(u / \nu)][\beta-(2 u / \nu)]^{-1} \exp \left[(u / \nu) \xi_{b}\right]}\right. \\
& \left.\times\left[\frac{\beta-(u / \nu)}{\beta-(2 u / \nu)} \beta \exp \left[(u / \nu) \xi_{b}\right]-\beta-(u / \nu)\right]-\frac{2 \exp \left(\beta \xi_{b}\right)}{\beta-(2 u / \nu)}+\frac{2}{\beta-(u / \nu)}+\chi^{2}(\nu / u)\right\} .
\end{aligned}
$$

These expressions give the value of dimensionless propagation velocity $u / \nu$ as a function of the principal variables and parameters of the process.

Expression (21) can be simplified by considering that $\exp \left(\beta \xi_{b}\right)=\phi\left(\xi_{b}\right)$ is small. Furthermore,

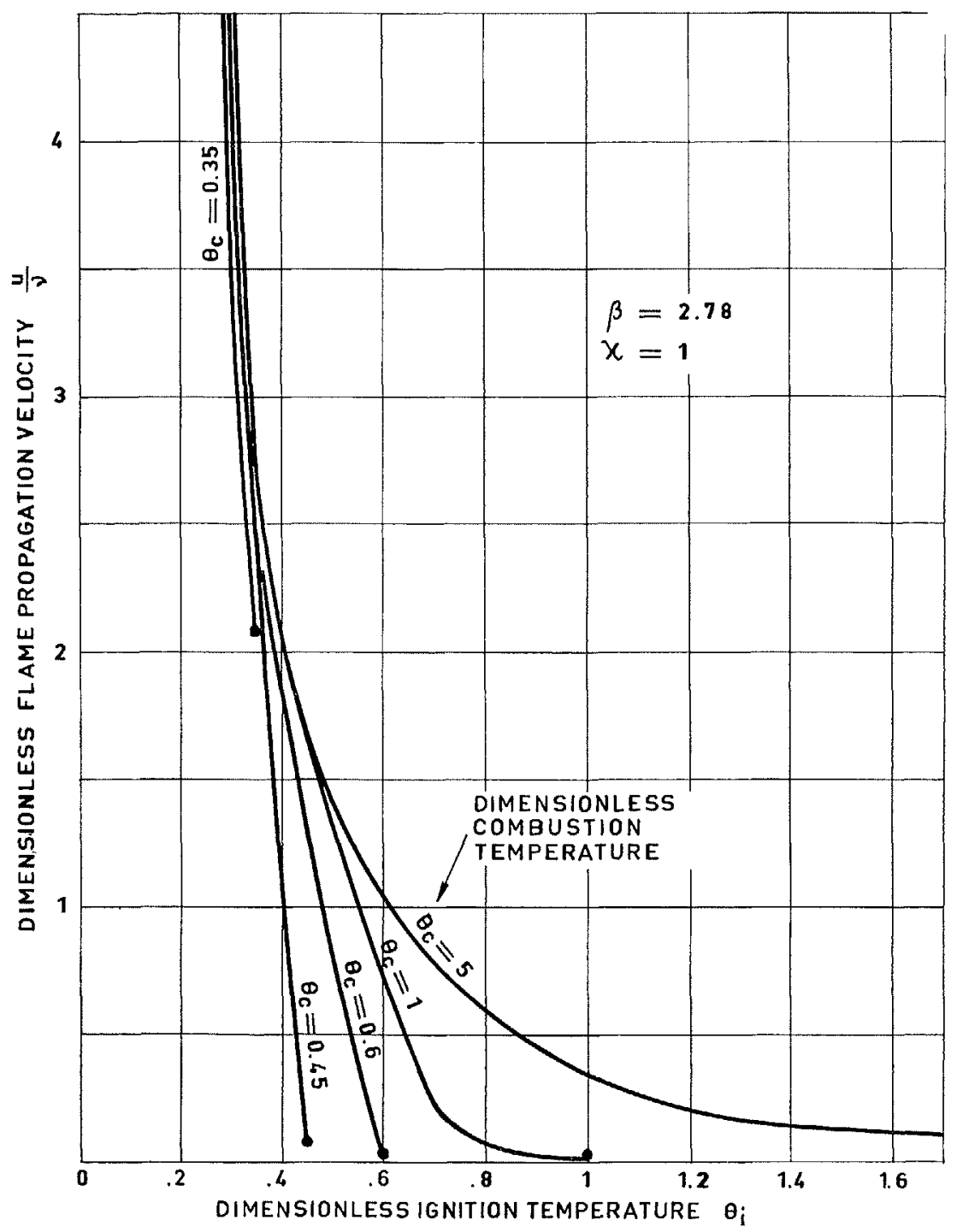

FIG. 2. Flame-propagation velocities as a function of ignition temperature. 
this boundary-layer approximation is only valid when the characteristic length of Eq. (1)

$$
l=\lambda / \rho c u
$$

is small as compared with any typical linear dimension of the process. Since

$$
(u / \nu) \xi_{b}=x_{b} /(\lambda / \rho c u)
$$

it is concluded that $(u / \nu) \xi_{b}$ must be large and then, $\exp \left[(u / \nu) \xi_{b}\right]$ is small (since $\xi_{b}<0$ ).

Introducing these simplifications into (21) results in

$$
\Psi=\chi^{-1}(\nu / u)\left[\frac{2}{\beta-(u / \nu)}+\chi^{2}(\nu / u)-\left(\theta_{i}^{2}+\frac{1}{\beta[\beta-(u / \nu)]}\right)[\beta+(u / \nu)]\right],
$$

which does not depend on $\xi_{b}$, meaning that flame-propagation speed does not depend on condition existing far ahead of the flame.

Figure 2 shows the values of $u / \nu$ as a function of the ignition temperature $\theta_{i}$ for several values of $\theta_{c}$. It may be seen that for small values of $\theta_{i}, u / \nu$ is large, combustion temperature $\theta_{c}$ does not influence the process, and the influence of convection is negligible. Results for $u / \nu \gg 1$ tend to those directly obtained from Eqs. (20) and (24), when condition $\chi=0$ is introduced, giving

$$
\frac{\nu}{u_{R}}\left[\frac{2}{\beta-\left(u_{R} / \nu\right)}-\left(\theta_{i}{ }^{2}+\frac{1}{\beta\left[\beta-\left(u_{R} / \nu\right)\right]}\right)\left[\beta+\left(u_{R} / \nu\right)\right]\right]-\left(\theta_{a}^{2}-\theta_{i}^{2}\right)=0,
$$

in which $u_{R}$ is the propagation velocity when radiant heat transfer is predominant.

On the other hand, if convective heat transfer is large as compared with radiant heat transfer, that is, $\chi \gg 1$, there is obtained

$$
\chi^{2} \frac{\nu^{2}}{u_{C}^{2}} \log \frac{\left(\nu^{2} / u_{C}^{2}\right) \chi^{2}}{\left(\nu^{2} / u_{c}^{2}\right) \chi^{2}-\theta_{i}}-\theta_{c}^{2}=0
$$

the expressions which gives directly the value of the propagation velocity $u_{C}$, when heat convection is the predominant heat transfer mechanism of the process.

\section{Flame Spreading over the Surface of A Liquid Fuel}

A theoretical and experimental application of the present study has been conducted for the case of flame spreading over the surface of hydrocarbon fuels burning in air.

Ignition temperature of the fuel $T_{i}$ was in this case the flashpoint temperature. Therefore, fuel initial temperatures were taken equal to or smaller than the flashpoint temperature, in order to have the flame-propagation mechanism studied in the present work.

This study was intended for rather large flames in which radiation heat transfer is predominant, even for steady combustion. ${ }^{8}$ For the case of flame propagation, a first study showed that the influence of convection was negligible, except perhaps, for very small propagation velocities.

Furthermore, since ignition temperature is not large as compared with initial temperature $T_{0}$ of the fuel, temperature gradients parallel to the fuel surface cannot be very large, and they were disregarded as compared with the high temperature gradients existing in a direction perpendicular to the fuel surface. For this condition, Eq. (9) reduces to

$$
\lambda\left(\partial_{2} T / \partial y^{2}\right)-\rho c u(\partial T / \partial y)=0
$$

an equation which has an exact analytical solution.

The fuel layer was supposed to be of finite thickness $y_{0}$ (or $\eta_{0}$ ), lying over a medium of thickness $y_{0}^{\prime}$ (or $\eta_{0}^{\prime}$ ). It was assumed that $y_{0}^{\prime}$ was large enough to be $(\partial T / \partial y)_{y=y_{0}}{ }^{\prime}=0$ throughout the propagation process.

Initial temperatures of fuel and supporting medium were taken both equal to $T_{0}$.

Radiant heat flux from the flame reaching the 
fuel surface, is given by expression

$$
Q_{0} q(x)=\epsilon \sigma T_{F}^{2} F=E F
$$

in which the total emissive power $E$ was experimentally determined.

By assimilating the shape of the flame to a rectangular surface of height $h$ and width $b$, geometrical factor $F$ was approximated by means of the expression ${ }^{2}$

$$
F=K \exp [\beta(x / \delta)]
$$

in which $K$ can be taken as constant and $\beta$ depends ${ }^{2}$ on the ratio $h / b$.

Under the preceding assumption, integration of Eq. (27) gives the following implicit expression for the flame-propagation velocity:

$$
\begin{array}{r}
{[\beta(u / \nu)]^{-1 / 2} \operatorname{coth}\left\{\eta_{0}[\beta(u / \nu)]^{1 / 2}+\Pi\right\}} \\
-\theta_{i}=0,
\end{array}
$$

in which function $\Pi$ is given by

$$
\tanh \Pi=\omega \tanh \left\{-\eta_{0}^{\prime} \omega\left[\beta(u / \nu]^{1 / 2}\right\}\right.
$$

where

$$
\omega=\left(\lambda^{\prime} \rho^{\prime} c^{\prime} / \lambda \rho c\right)^{1 / 2}
$$

This solution is valid, provided that the parameter

$\Lambda=\left|\omega \tanh \left\{-\eta_{0}^{\prime} \omega[\beta(u / \nu)]^{1 / 2}\right\}\right|<1$.

On the other hand, if $\Lambda>1$, the following solution appears

$[\beta(u / \nu)]^{-1 / 2} \tanh \left\{\eta_{0}[\beta(u / \nu)]^{1 / 2}+\Pi\right\}$

$$
-\theta_{i}=0
$$

being

$$
\operatorname{coth} \Pi=\omega \tanh \left\{-\eta_{0} \omega[\beta(u / \nu)]^{1 / 2}\right.
$$

Flame-propagation velocities are shown in Fig. 3 as function of fuel thickness $\eta_{0}$. As it might be expected, above a certain value of $\eta_{0}$, flamepropagation velocity does not depend on it. On the other hand, flame-propagation velocity increases as the fuel thickness augments it if $\omega>1$, and conversely.

Temperature profiles aro represented in Fig. 4. They were obtained with the expression

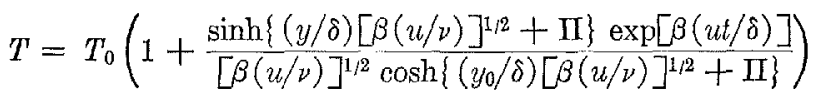

in which $\eta$ is given by $(35)$

It may be observed that temperature gradients are very strong in the vicinity of the fuel surface, confirming the assumption previously made.

\section{Experimental Results}

The first part of the experimental work was devoted to the study of the possibility of having a steady flame-propagation process in a channel, as well as to the study of the influence of both channel width and flame length on the process.

This program was accomplished with the research facility shown in Fig. 5.

Flame size was controlled by means of a folding screen or sheet-metal train moving behind the flamefront and at the same speed. This screen quenches the flame as it progresses, keeping both flame size and propagation velocity constant,
It was observed that flame propagation speed does not depend on channel width above a certain value, and that this speed increases as the flame depth augments it until a maximum value is reached.

If the folding screen was not used, a reasonable constant propagation velocity was obtained, if such speed was measured after the flame had propagated through one-third of the channel length.

According to these results, and in order to study the influence of fuel depth and fuel initial temperature, a new larger channel was prepared, 16 feet in length and 20 in. width. The channel was filled with water heated to the desired initial temperature, and then the fuel was spread over the water.

Ignition was achieved with the help of small amount of gasoline, and propagation velocities were measured by means of a movie camera or by using a chronometer. 


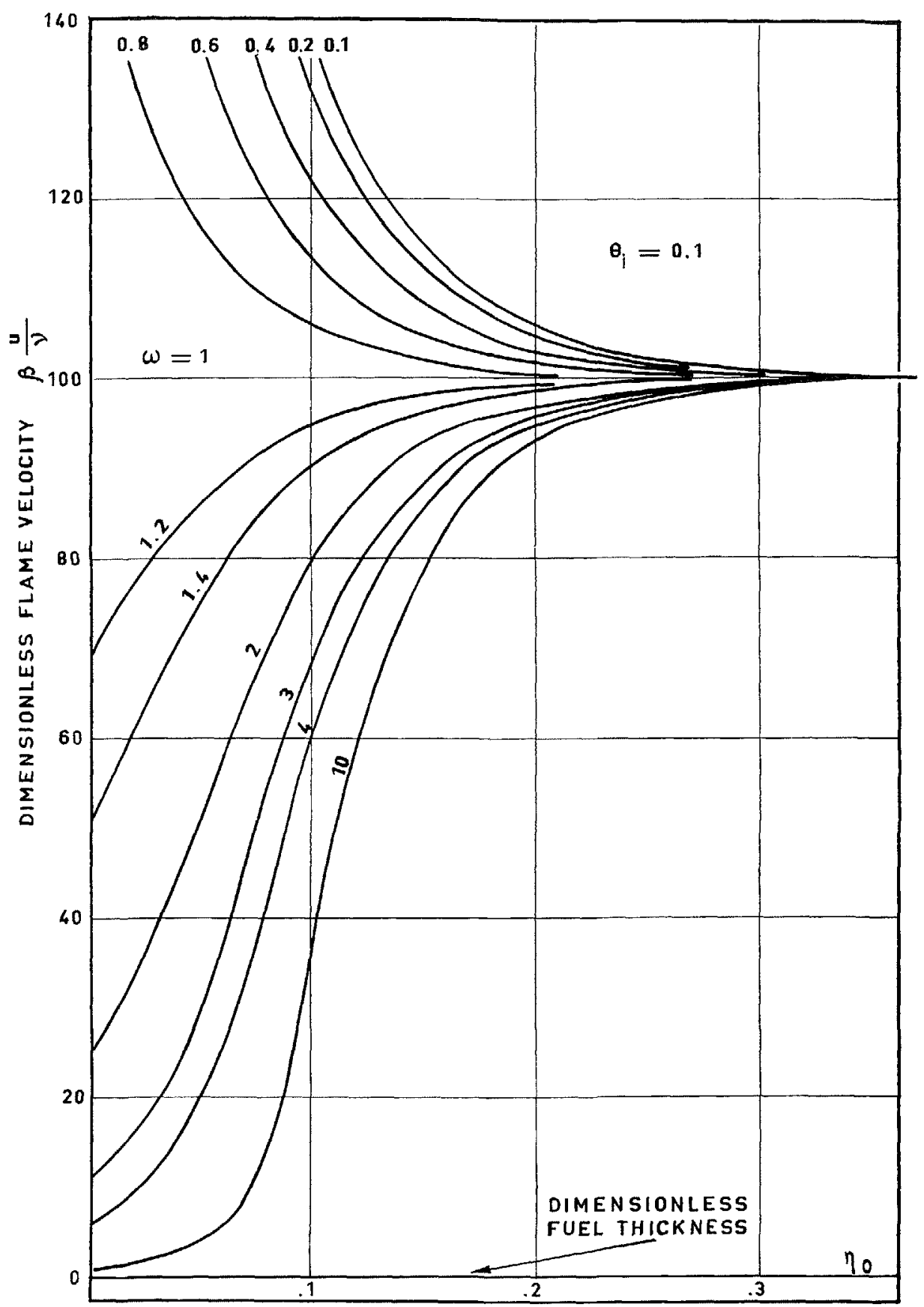

Fig. 3. Flame spreading over the surface of a liquid fuel. Flame velocities as a function of fuel depth. 


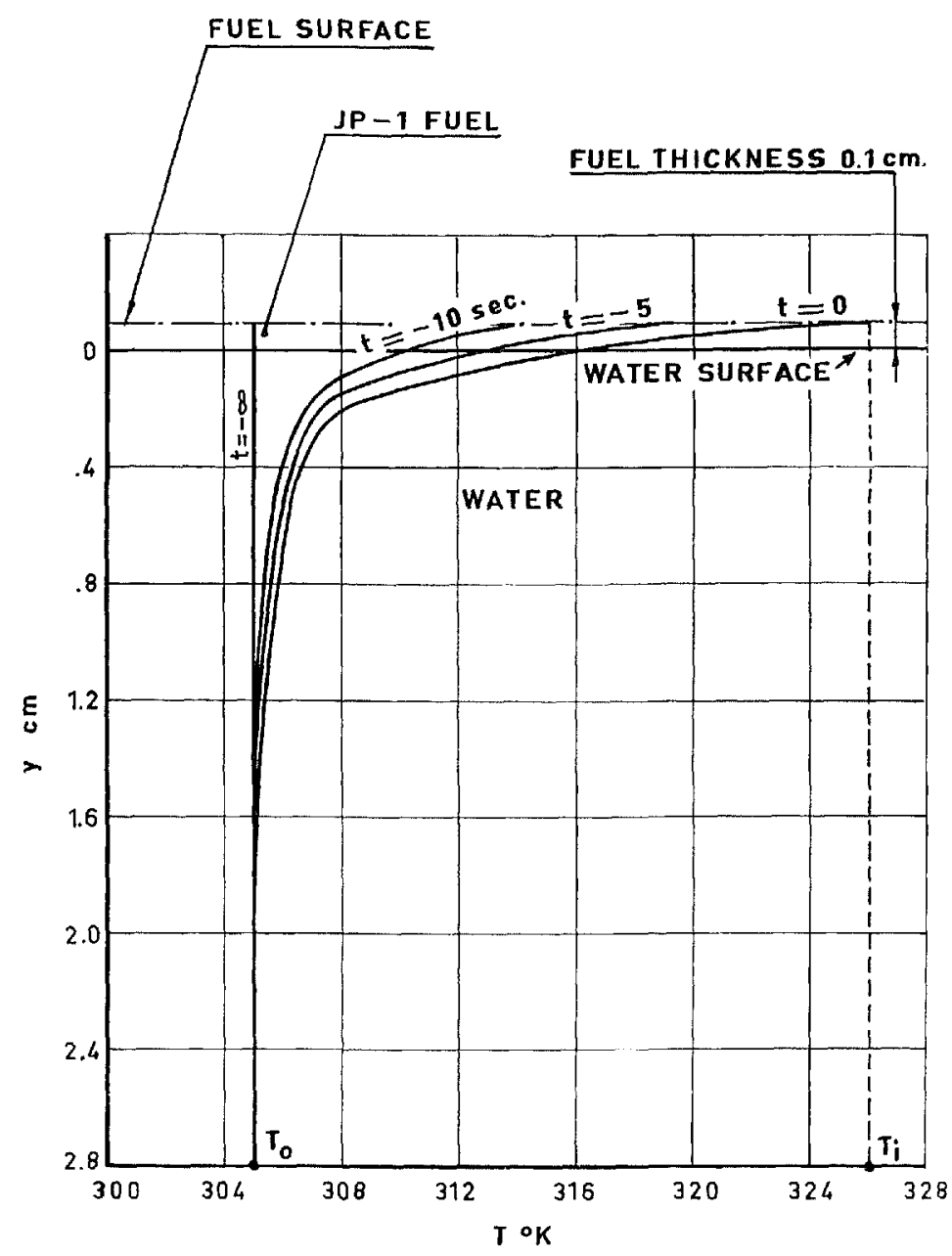

Fig. 4. Flame spreading over the surface of a liquid fuel. Theoretical temperature profiles.

Some typical experimental results are shown in Fig. 6.

By measuring the flame emissive power by means of radiometers it was possible to calculate the flame-propagation speed using expressions (34) and (35). (These measurements were carried out in a steady flame of equal size.)

In Fig. 6 a comparison between theoretical and experimental results is also included. It may be seen that they agree reasonably well.

For high propagation velocities, when fuel initial temperature is close to ignition temperature, theoretical values are higher than those experimentally obtained. This result could be expected, since for $T_{0}=T_{i}$ the theoretical propagation velocity given by this model is infinite. Actually, at temperatures equal or higher than flashpoint temperature, flame propagates through the combustible gaseous mixture already existing over the fuel surface.

On the other hand, at low propagation velocities, theoretical results are smaller than experimental values, which is probably due to the influence of convective heat transfer, which may exert some influence in the region of very low propagation velocities.

\section{Nomenclature}

C specific heat

$E \quad$ emissive power 


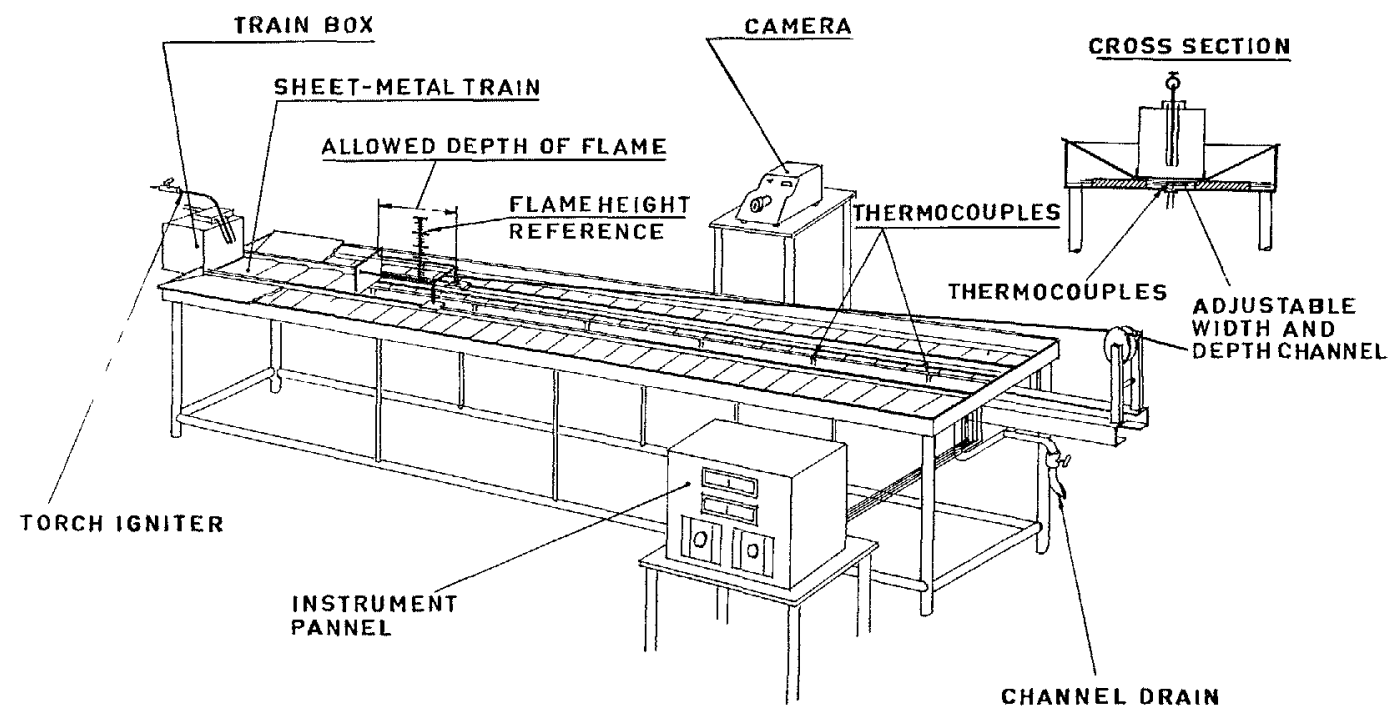

FIG. 5. Flame spreading over the surface of a liquid fuel. Research facility.

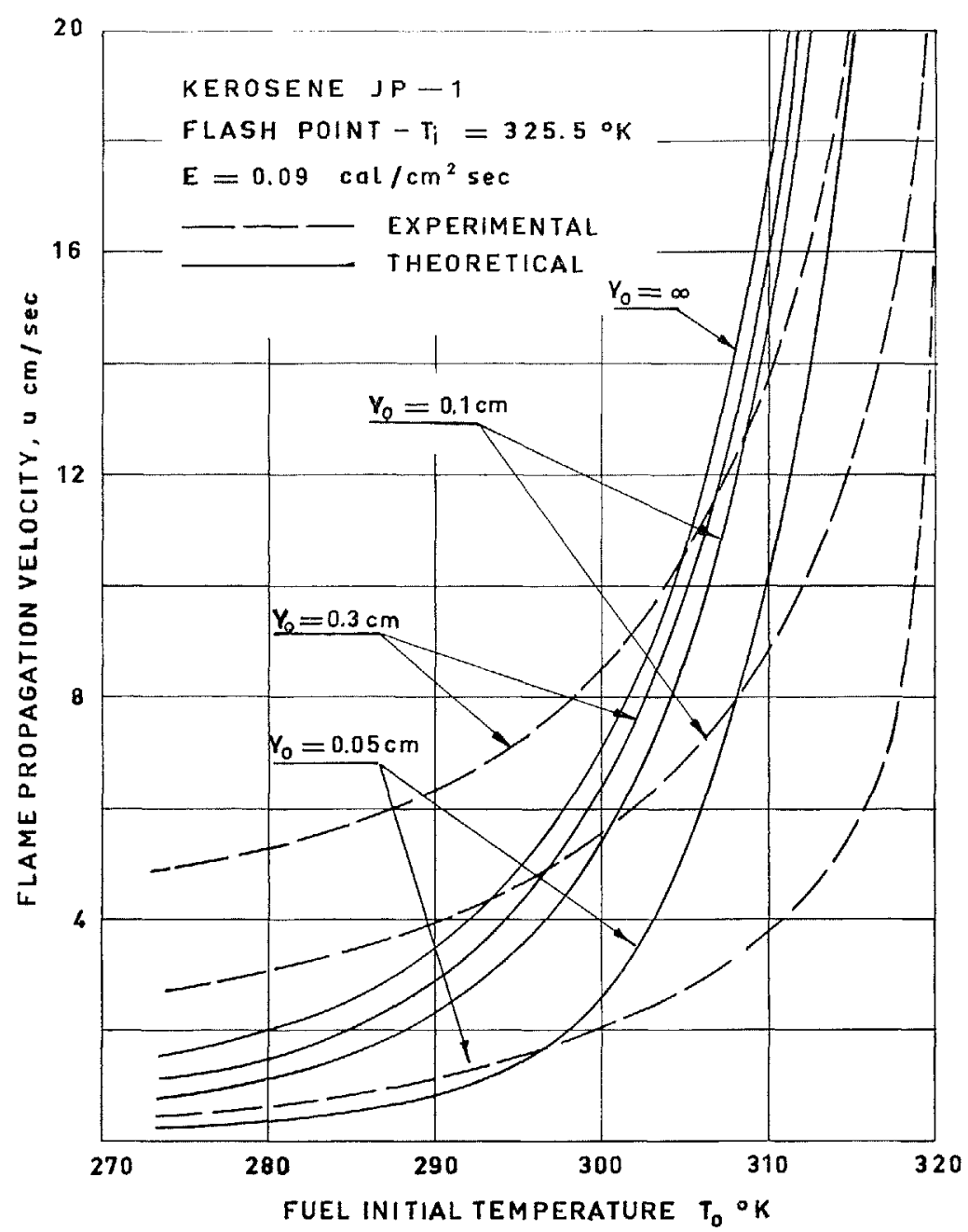

Fig. 6. Flame spreading over the surface of JP-1 fuel. Comparison between theoretical and experimental results. 


$\begin{array}{ll}F & \text { geometrical factor. } \\ l & \text { characteristic length } \\ K & \text { parameter } \\ \dot{m} & \text { evaporation rate } \\ \mathrm{Pr} & \text { Prandt Number } \\ q_{1} & \text { latent heat of vaporization } \\ t & \text { time } \\ T & \text { temperature } \\ u & \text { flame propagation velocity } \\ \beta & \text { parameter } \\ \delta & \text { characteristic length } \\ \epsilon & \text { flame emissivity } \\ \eta & \text { dimensionless coordinate perpendicu- } \\ & \text { lar to the fuel surface } \\ \eta_{0} & \text { dimensionless thickness } \\ \theta & \text { dimensionless temperature } \\ \lambda & \text { thermal conductivity } \\ \nu & \text { characteristic velocity, also kinematic } \\ \xi & \text { viscosity } \\ \Phi & \text { dimensionless coordinate parallel to } \\ \Phi & \text { fuel surface } \\ \Psi & \text { function } \\ \Pi & \text { function } \\ \rho & \text { function } \\ \sigma & \text { density } \\ \omega & \text { Stefan-Boltzmann constant } \\ Q_{0} \phi(\xi) & \text { parameter } \\ Q_{0} q(\xi) & \text { heat flux through the fuel surface } \\ Q_{0} \chi(\xi) & \text { ahead flux through the fuel surface } \\ & \text { heat flux through the fuel surface } \\ & \text { behind the flame } \\ & \\ & \end{array}$

Subscripts

$\begin{array}{ll}c & \text { combustion } \\ g & \text { gas phase } \\ i & \text { ignition } \\ o & \text { initial conditions. }\end{array}$

Superscripts

$$
\text { supporting medium }
$$

ACKNOWLIEDGMENT

The research reported in this document has been sponsored by the Air Force Office of Scientific Research under Grant AF EOAR 63-44 through the European Office of Aerospace Research (OAR), U.S. Air Force.

\section{REFERENCES}

1. Emmons, H. W.: Tenth Symposium (International) on Combustion, The Combustion Institute, 1965.

2. Sanchez Tarifa, C., et al.: Heterogeneous Combustion and Flame Structures, Grant AF EOAR 63-44, Final Report, INTA, Madrid, 1965.

3. MCAlevy, III, R. F., Magee, R. S., AND Wruber, J. A.: AIAA Solid Propellant Rocket Conference, Palo Alto, California, January 1964.

4. Desoto, S. and Frigdman, H. A.: AIAA J. $8,405(1965)$

5. Horowiri, F. A.: Stevens Institute of Technology Report, June 1965.

6. Hicks, B. L.: J. Chem. Phys. 2Q, 414 (1954).

7. McAlevy, III, R. F.; Conan, P. L., and Summerifietd, M.: Solid Propellant Rocket Research, Vol. 1, Academic Press, 1960.

8. Sanchez Tarifa, C., et al.: Open Fires and Transport of Firebrands, U.S. Forest Service Grant FG-Sp-114. Second and Third annual Reports, INTA, Madrid, 1963-1964.

\section{COMMENTS}

Dr. A. F. Roberts (Safety in Mines Research Establishment, Buxton): In a series of experiments in which the spread of flame across the surface of iso-amyl alcohol was studied, I measured the surface temperature at the base of the spreading flame and obtained the same result as Dr. Tarifa, namely that this temperature was close to the liquid's flash point. Being an alcohol Hame, however, no significant radiative heat transfer took place. By using a transparent vessel and schlieren techniques for visualizing flow patterns in the liquid, I was able to observe a current of hot liquid of depth $<0.1 \mathrm{~mm}$, spreading over the surface of the liquid ahead of the flame. The distance between the tip of this current and the flame remained substantially constant and heat transfer to the liquid was most intense at the base of the flame.
Using this information, I developed a mathematical model of the process in which the flame was represented by a strip source of heat moving across the surface of the liquid, in a direction perpendicular to its length, and heat transfer within the liquid was represented by the laws of heat conduction, using "effective thermal conductivities" which were different for the horizontal and vertical directions. The experimentally observed criterion for the flame spreading to a particular region was used in this model.

Using a single set of values for the effective thermal conductivities gave good agreement between calculated and observed rates of spread, and between calculated and observed temperature variations over a wide range of conditions. (Roberts, A. F.: Ph.D. thesis, University of Lon- 
don, 1966, "The Spread of Flame across Liquid Surfaces.")

Professor Spalding raised the question of the extent to which a "critical temperature" criterion was useful in this sort of work as the concept of laminar-flame theory.

The "critical temperature" for the spread of flame across a liquid surface is the flash point of the liquid. The flash point of a liquid, as determined in a stirred closed system, has been shown experimentally to be that temperature at which the equilibrium vapor pressure of the liquid is such that the resulting vapor/air mixture has the same composition as a mixture at the lower inflammability limit, as measured in the ordinary way. This being so, when a liquid's surface temperature is raised locally to its flash point, a layer of vapor/air mixture is produced adjacent to the surface which is within the inflammability limits of the vapor. The thickness of this layer will depend on masstransfer consideration in the vapor phase.

Therefore, the critical temperature used in this work for liquid is not directly comparable to a chemically controlled ignition temperature. It is more closely related to the concept of limits of inflammability.

Dr. C. Sanchez Tarifa: In the paper we explained in what way the fuel-ignition temperature has to be considered. The fuel heated by the flame vaporizes, and these vapors mix with the gaseous oxidizer until a combustible mixture forms ahead of the flamefront, through which the flame-spreading process takes place.

Fuel-vapor mixing is controlled by the diffusion equations in which the fuel mass flow evaporated per unit time enters both as a convective term and in the boundary conditions of the problem. This mass flow is a very sensitive function of the fuelsurface temperature and, for liquids (under boiling temperature), it can be expressed as an exponential function of that temperature.

Flame spreading is conditioned to the existence of a minimum concentration of fuel vapors ahead of the flame, and this minimum concentration can be directly related to the fuel-surface temperature through the diffusion equations. Therefore, there exists a local fuel-surface temperature for which flame spreading occurs, and this temperature has to be considered as the "ignition temperature" of the process.

The problem would have been quite different if forced convection of the gaseous oxidizer would have been considered, because, in this case, fuelvapor concentration ahead of the flamefront would depend not only on fuel-surface temperature but also on the aerodynamic field. 